\title{
Correction to: Osseoperception in transcutaneous osseointegrated prosthetic systems (TOPS) after transfemoral amputation: a prospective study
}

\author{
Marcus Örgel ${ }^{1} \mathbb{D}$ - Mohamed Elareibi ${ }^{1}$. Tilman Graulich ${ }^{1} \cdot$ Christian Krettek $^{1}$ - Claudia Neunaber ${ }^{1}$. \\ Horst-Heinrich Aschoff ${ }^{1} \cdot$ Alexander Ranker $^{2} \cdot$ Marcel Winkelmann ${ }^{1}$
}

Published online: 17 December 2021

(c) Springer-Verlag GmbH Germany, part of Springer Nature 2021

\section{Correction to: Archives of Orthopaedic and Trauma Surgery https://doi.org/10.1007/s00402-021-04099-1}

The original version of this article unfortunately contained a mistake. In Table 4, Männlich and Weiblich should be changed into male and female.

The corrected Table 4 is placed in the following page.

The original article can be found online at https://doi.org/10.1007/ s00402-021-04099-1.

Marcus Örgel

Oergel.Marcus@mh-hannover.de

1 Department of Trauma, Hannover Medical School (MHH),

Carl-Neuberg-Straße 1, 30625 Hannover, Germany

2 Department of Physical Medicine and Rehabilitation,

Hannover Medical School (MHH), Carl-Neuberg-Str. 1,

30625 Hannover, Germany 
Table 4 Presentation of the subgroup analysis divided according to gender

\begin{tabular}{|c|c|c|c|}
\hline & Male $(n=46)$ & Female $(n=29)$ & $p$ value \\
\hline \multicolumn{4}{|l|}{ Amputation side $n(\%)^{\# \#}$} \\
\hline Right & $27(58.7)$ & $16(55.2)$ & \multirow[t]{2}{*}{$0.8^{\mathrm{a}}$} \\
\hline Left & $19(41.3)$ & $17(44.8)$ & \\
\hline \multicolumn{4}{|l|}{ Reason for amputation $n(\%)$} \\
\hline Trauma & $26(56.5)$ & $10(34.5)$ & \\
\hline Vascular & $5(10.9)$ & $4(13.8)$ & \\
\hline Tumor & $3(6.5)$ & $2(6.9)$ & \\
\hline No amputation & $12(26.1)$ & $13(48.2)$ & \\
\hline Age mean $\pm \mathrm{SD}, 95 \% \mathrm{CI}$, median $(\mathrm{IQR})^{\#}$ & $52.2 \pm 12.0,48.0-56.4,51.5(15)$ & $49.8 \pm 9.7,44.6-54.9,50(12)$ & $0.05^{\mathrm{b}}$ \\
\hline Years since amputation mean $\pm \mathrm{SD}, 95 \% \mathrm{CI}$, Median $(\mathrm{IQR})^{\#}$ & $15.9 \pm 11.4,11.9-19.9,16(16)$ & $16.4 \pm 11.5,10.3-22.5,15.5(19)$ & $0.9^{\mathrm{b}}$ \\
\hline $\mathrm{BMI}\left[\mathrm{kg} / \mathrm{m}^{2}\right]$ mean $\pm \mathrm{SD}, 95 \%-\mathrm{CI}$, median $(\mathrm{IQR})^{\#}$ & $26.5 \pm 4.0,25.2-27.9,26.3(5)$ & $28.5 \pm 5.4,25.6-31.4,27.4(6)$ & $0.7^{\mathrm{b}}$ \\
\hline $\mathrm{V} 1$ mean $\pm \mathrm{SD}, 95 \% \mathrm{CI}$, median $(\mathrm{IQR})^{\# \#}$ & $2.0 \pm 1.3,1.6-2.5,2.0(2)$ & $1.7 \pm 1.1,1.1-2.3,2(1)$ & $0.9^{\mathrm{a}}$ \\
\hline $\mathrm{V} 2$ mean $\pm \mathrm{SD}, 95 \% \mathrm{CI}$, median $(\mathrm{IQR})^{\# \#}$ & $1.4 \pm 1.1,1.0-1.7,1.0(2)$ & $1.3 \pm 1.3,0.7-2.0,1(3)$ & $0.6^{\mathrm{a}}$ \\
\hline $\mathrm{V} 3$ mean $\pm \mathrm{SD}, 95 \% \mathrm{CI}$, median $(\mathrm{IQR})^{\# \#}$ & $2.9 \pm 0.3,2.8-3.1,3.0(0)$ & 3 (constant) & $0.4^{\mathrm{a}}$ \\
\hline Total V1-V3 mean $\pm \mathrm{SD}, 95 \%-\mathrm{CI}$, median $(\mathrm{IQR})^{\#}$ & $6.2 \pm 1.7,5.6-6.8,6(3)$ & $6.0 \pm 1.8,5.1-7.0,6(4)$ & $0.5^{\mathrm{b}}$ \\
\hline $\mathrm{PMQ}$ mean $\pm \mathrm{SD}, 95 \% \mathrm{CI}$, median $(\mathrm{IQR})^{\# \#}$ & $24.4 \pm 21.0-27.8,25.0(17)$ & $27.5 \pm 9.3,22.3-32.6,29.0(18)$ & $0.3^{\mathrm{b}}$ \\
\hline $\mathrm{K}-$ Level mean $\pm \mathrm{SD}, 95 \% \mathrm{CI}$, median $(\mathrm{IQR})^{\#}$ & $2.6 \pm 0.7,2.3-2.9,3.0(1)$ & $2.6 \pm 0.8,2.13 .1,3.0(1)$ & $0.6^{\mathrm{a}}$ \\
\hline Prosthesis wear time mean $\pm \mathrm{SD}, 95 \% \mathrm{CI}$, median $(\mathrm{IQR})^{\#}$ & $11.1 \pm 6.1,9.0-13.2,11.0(7)$ & $11.1 \pm 5.3,8.1-14.0,14.0(7)$ & $0.8^{\mathrm{a}}$ \\
\hline
\end{tabular}

\#Normally distributed data

\#\# Non-normally distributed data

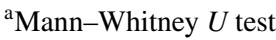

${ }^{\mathrm{b}} T$ test

Publisher's Note Springer Nature remains neutral with regard to jurisdictional claims in published maps and institutional affiliations. 